Ann. Sci. forest., 1982, 39 (1), 33-40

\title{
Estimation de l'utilité d'une introduction d'aulne glutineux en mélange à de jeunes plants d'épicéa commun sur sol à hydromorphie temporaire superficielle
}

\author{
G. LEVY \\ avec la collaboration technique de Y. LEFìvrE \\ I.N.R.A.. Station de Recherches sur les Sols forestiers ot la Fertilisation \\ Centre de Recherches forestières de Nancy, \\ Champenoux, F 54280 Seichamps
}

\begin{abstract}
Résumé
T'expérience dont il est rendu compte a pour objectif d'estimer l'efficacité éventuelle d'un drainage "biologique» du sol par l'aulne glutineux, introduit en mélange avec l'essence principale, l'épicéa en l'occurrence.

Elle s'est déroulée en milieu contrôlé, pendant deux ans, sur des plants âgés de 2 ans au début de l'expérience. La nappe du témoin (sans aulne) est maintenue proche de la surface par des apports périodiques d'eau, un volume identique étant versé dans les bacs contenant le mélange d'espèces. La composition testée est de un aulne pour cinq épicéas.

Il est possible de souligner les résultats suivants :

- l'aulne abaisse la nappe d'une manière efficace (ćvapotranspiration moyenne augmentée de 19 à 34 p. 100 selon les conditions), et cela dès la reprise annuelle d'activité de l'épicéa ;

- cet abaissement provoque des effets favorables sur le comportement de l'épicéa (enracinement, nutrition azotée, survie) ;

- l'aulne résiste à un certain dessèchement du sol en été ; il provoque cependant un accroissement de co dessèchement estival qui pourrait être préjudiciable à l'épicéa dans certaines conditions de station, bien que l'enracinement de cette dernière espèce, et par conséquent le volume de sol prospecté, soient fortement augmentés grâce à la présence de l'aulne.
\end{abstract}

\section{I. - Introduction}

L'épicéa commun est très utilisé pour le reboisement des sols à hydromorphie temporaire (pseudogleys) notamment dans l'Est de la France.

Il s'enracine cependant très mal dans les horizons marmorisés (Delvaux, 1968) et sa production finale est très affectée dans les stations où la nappe remonte 
jusquà proximité de la surface du sol (Levy, 1978). Ce type de nappe est déjà très néfaste à l'enracinement, la nutrition azotée, la survie et la croissance de jeunes plants, comme l'ont montré des essais en milieu contrôlé (LEvy, 1971 et 1981); un assainissement du sol pourrait donc éventuellement permettre, outre un accroissement de la productivité de la station, un bon démarrage des plantations, done une réduction des frais dentretien.

Des expériences (notamment LEvy, 1972) ont montré l'efficacité du drainage sur la croissance initiale de lépicéa dans certairess stations; mais des fossés à faible écartement $(20 \mathrm{~m}$ ou même moins) sont nécessaires, ce qui créc d’importantes contraintes financières et matérielles. Il scrait donc sans doute fort intéressant de réaliser un assainissement biologique, en introduisant, en mélange à l'épicéa, une espèce ligneuse qui résiste à un engorgement superficiel du sol et abaisse le niveau de la nappe.

Nous avoas persé à cette fin à laulne glutineux, que lon rencontre dans des sols à nappe souvent superficielle à proximité de ruisseaux ou d'étangs. Il peut survivre dans un milieu virtuellement dépourvu d’oxygène (Braun, 1974); sa grande tolérance à ces conditions défavorables est la conséquence dadaptations, telle la formation de racines adventives prenant naissance sur des lenticelles hypertrophiées (GıLl, 1970 et 1975). Cependant, croissant généralement sur des sols à nappe permanente, supporterait-il le dessèchement périodique des pseudogleys? On peut a priori être relativement optimiste à ce sujet : Krrutzer (1961) a en effet montré que laulne glutineux avait un enracinement remarquablement profond et dense dans les pseudogleys; il est donc susceptible de prélever de l'eau dans un important volume de sol. D'ailleurs, cette espèce est déjà utilisée, en mélange à l'essence principale, soit comme culture d'abri, afin d’améliorer le microclimat (par exemple, FERDA, 1973), soit comme améliorateur de la nutrition sur sol pauvre, en raison de son pouvoir de fixer l'azote atmosphérique grâce aux nodosités de ses racines (par exemple Hausser, 1973, ou HEINSDORF, 1974).

Nous avons done voulu, d'une part et surtout, vérifier si l'aulne glutineux introduit en mélange à l'épicéa était susceptible dabaisser sensiblement le niveau dure nappe temporaire superficielle, d'autre part suivre l'évolution du dessèchement du sol par laulne et la résistance de cette espèce à de faibles potentiels hydriques après disparition des nappes.

\section{II. - Matériel et méthodes}

S’il apparaît utile, pour se faire une idée assez précise de lintérêt de l'aulne, d'opérer en milieu contrôlé, les récipients utilisés ne devaient cependant pas avoir des dimensions trop réduites, afin de réaliser un modèle se rapprochant des conditions naturelles.

Les conditions expérimentales, notamment la dimension des plants, la proportion relative des individus des deux espèces, les niveaux entre lesquels la nappe fluctue, ont été choisis un peu arbitrairement, mais elles permettent d'estimer l'ordre de grandeur des phénomènes qui nous intéressent.

L'expérience a été menée dans quatre bacs en béton étanche de $0,70 \mathrm{~m}$ dans les trois dimensions. Ces récipients ont été remplis d'une terre provenant d'un mélange des horizons $A_{1}$ et $A_{2}$ d’un sol brun lessivé à pseudogley (provenant de la forêt 
domaniale d'Amance), bien représentatif, notamment par sa texture limono-argileuse, des zones de battement de la nappe temporaire de nombreux pseudogleys. Les quatre bacs sont disposés deux par deux, chacun des deux groupes constituant un bloc; le sol du bloc 1 est finalement plus tassé que celui du bloc 2 : ne provenant pas exactement du même endroit, en forêt, il est légèrement moins organique, et sa structure est donc moins stable; à la fin de l'expérience, entre -8 et $-12 \mathrm{~cm}$ dans les bacs sans aulne, le coefficient Is d'instabilité structurale est ainsi égal à 1,73 dans le bloc 1, 0,98 dans le bloc 2 .

Deux piezomètres ont été installés dans chaque bac, afin notamment de mesurer le niveau des nappes et d'effectuer les apports d'eau. L'expérience comporte deux traitements. Dans les bacs appartenant au premier d'entre eux ont été repiqués uniquement 36 épicéas communs 2-0 de provenance Velay-Maygal. Dans les deux autres bacs $(2 *$ traitement $), 6$ aulnes glutineux répartis à peu près uniformément remplacent 6 épicéas : ce traitement comporte donc 30 épicéas et 6 aulnes.

L'expérience a duré deux ans.

Au cours de la première année, une nappe a été installée à $-5 \mathrm{~cm}$ dans tous les bacs un moins environ après le repiquage. Des apports ultérieurs d'eau ont été effectués pendant un mois, par bloc : lorsque la nappe s'était abaissée jusqu'à - 15 à - $20 \mathrm{~cm}$ dans le bac sans aulnes, on ramenait son niveau à $-5 \mathrm{~cm}$, et un même volume d'eau était versé dans le bac contenant des aulnes. Le sol s'est ensuite ressuyé, puis s'est relativement desséché au cours de l'été ; un même volume d'eau était cependant apporté périodiquement dans les deux bacs d'un même bloc, afin d'éviter une trop forte sécheresse. Les aulnes ont été taillés au cours de l'été pour empêcher notamment un ombrage des épicéas.

Fin janvier de la seconde année, une nappe a été installée en surface dans les 4 bacs; puis elle était ramenće en surface, dans chacun des bacs sans aulne, chaque fois qu'elle était redescendue jusqu'à $-8 \mathrm{~cm}$ (en réalité parfois jusqu'à une plus grande profondeur), et un même volume d'eau était versé dans le bac «à aulnes » du même bloc.

Le ressuyage a débuté le 4 juin. Au cours de l'été, le sol était réhumecté par engorgement de quelques heures simultanément dans les deux bacs d'un même bloc lorsque le $\mathrm{pF}$ à $-14 \mathrm{~cm}$ (mesuré d'une façon imprécise à l'aide de sondes «Gély») atteignait environ 4,0 dans le bac qui se desséchait le plus, c'est-à-dire celui contenant des aulnes. Les aulnes ont été tailiés à plusieurs reprises, le 2 avril pour la première fois.

Des mesures de pousses et des comptages de mortalité ont été effectués à plusieurs reprises. Les systèmes racinaires des plants ont été examinés en novembre de la $2^{\mathrm{e}}$ année de l'expérience.

\section{III. - Résultats}

\section{1. - Première année de l'expérience}

Cette première année est beaucoup moins intéressante que la seconde car la nappe n'est guère néfaste à l'épicéa en raison des caractéristiques de ses fluctuations (elle fluctue entre $-5 \mathrm{~cm}$ et $-20 \mathrm{~cm}$ ) et de sa faible durée, et d'autre part les $\mathrm{pF}$ du sol au cours de l'été n'ont pas été relevés. On ne constate d'ailleurs aucun effet négatif de la nappe sur les épicéas. 
Il est cependant déjà possible d'avoir une idée de l'abaissement de nappe provoqué par l'aulne. Ainsi, dans le bloc 1, après trois semaines d'engorgement et deux apports successifs d'eau, identiques dans les deux bacs, et dont la valeur cumulée se monte à $11 \mathrm{~mm}$, la nappe est à $-20 \mathrm{~cm}$ dans le bac sans aulne et à $-35 \mathrm{~cm}$ dans l'autre bac. Si l'on ramène alors la nappe à $-5 \mathrm{~cm}$, on s'aperçoit qu'en 3 semaines il y a eu une évapotranspiration de $17,6 \mathrm{~mm}$ d'eau dans le bac sans aulne et approximativement de $6 \mathrm{~mm}$ en plus dans l'autre bac : l'évapotranspiration a donc été supérieure d'environ 34 p. 100 dans le bac contenant des aulnes.

\section{2. - Seconde année de l'expérience}

\subsection{1. - Fluctuation des nappes}

La figure 1 représente, à titre d'exemple, pour le bloc 2, la variation en fonction du temps, d'une part du niveau de la nappe du bac sans aulne, d'autre part de la différence entre les niveaux des nappes dans les deux bacs avant l'apport (celui contenant des aulnes ayant toujours la nappe la plus profonde).

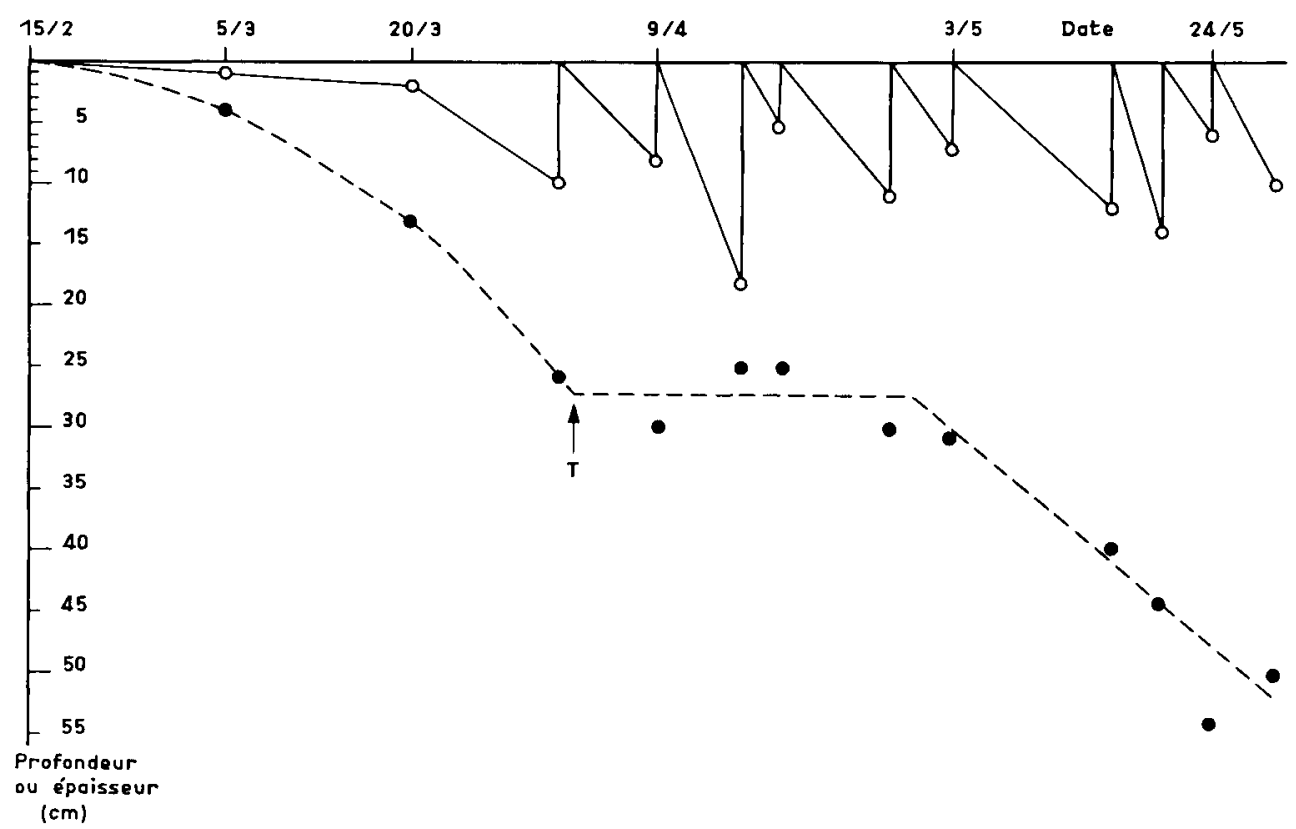

Fig. 1

Influence d'un mélange d'aulne sur labaissement de la nappe par rapport ì l'épicéa pur (addition périodique d'un même volume d'eau dans les deux traitements)

Influence of an Alder-Spruce mixture on the water-table lowering, related to alone Sprace (periodical supply of a same water volume in both treatments)

Légende :

Prolondeur de la nappe dans le bac sans aulne.

Water-table deepness in without Alder vat.

- - Différence d'épaisseur de nappes entre bacs avec et sans aulnes.

Water-table thickness difference between with and without Alder vats. 
On constate ainsi que le 24 mai cette différence est devenue très importante, les nappes se trouvant respectivement à $-6 \mathrm{~cm}$ et $-60 \mathrm{~cm}$.

La différence d'épaisseur des deux nappes, ou, ce qui revient au même, leur différence de niveau, augmente à peu près uniformément dans le temps, sauf au cours du mois d'avril, où elle reste à peu près stable: l'aulne na alors aucun effet sur le niveau de la nappe. On suppose que ceci est la conséquence d'une taille assez sévère, la première de l'année, qu’ont subi les aulnes le 2 avril. Les tailles ultérieures, moins importantes, sont au contraire sans effet sur la transpiration des aulnes.

Les résultats sont globalement comparables pour le premier bloc, notamment la différence finale de niveau de nappe entre les deux traitements. Laulne ne commence cependant à abaisser sensiblement la nappe, dans ce bloc, qu’à partir de la troisième décade de mars.

Pour l'ensemble de la période d'apport d'eau, c'est-à-dire du 15 février au 24 mai, le bac sans aulne du 2" bloc a évapotranspiré $110,8 \mathrm{~mm}$ deau. La consommation supplémentaire d'cau dans le bac contenant des aulnes a élé d'environ $31 \mathrm{~mm}$ d'eau, c'est-à-dire de 28 p. 100.

Dans le premier bloc, dont le sol est plus tassé, cette dernière valeur est voisine de 19 p. 100 , alors qu'elle était de 34 p. 100 au cours de la première année ; plusieurs causes peuvent expliquer cette différence, en particulier la taille des aulnes effectuée le 2 avril de la seconde année.

\subsection{2. - Dessèchement estival du sol}

Au début de cette période, l'humidité du sol est plus importante dans les bacs sans aulne car la nappe y était bien plus superficielle juste avant le ressuyage.

Le 11 juillet, on note les $\mathrm{pF}$ moyens suivants :

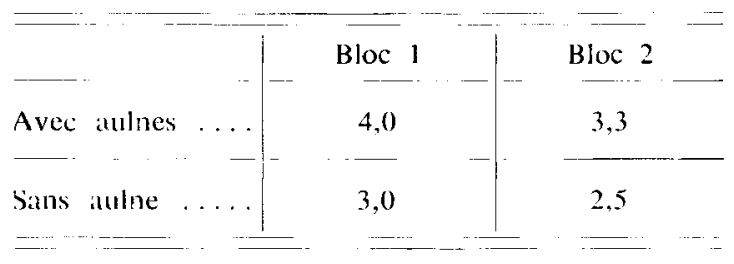

Le premier bloc s'est donc desséché plus rapidement que le second, probablement en raison du tassement plus important de son sol. Ce bloc est alors réhumecté par engorgement d'une demi-journée ; le lendemain, les $\mathrm{pF}$ y sont respectivement de 3,3 et 2,8 pour les deux traitements.

Le 16 août, les $\mathrm{pF}$ sont les suivants :

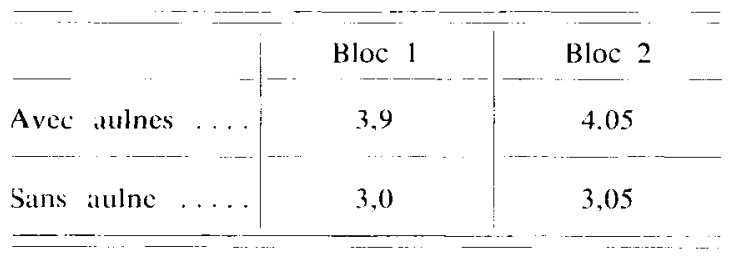

Il apparaît donc évident que les aulnes ont accéléré le dessèchement du sol. 


\subsection{3. - Comportement des épicéas}

\section{- Mortalité et croissance}

Aucun dépérissement n'est apparu au cours de la première année de l'expérience. Ce n'a pas été, par contre, le cas l'année suivante; ainsi, au début de l'été, on enregistrait, en moyenne pour les deux blocs, 62 p. 100 de plants morts ou dépérissants dans les bacs sans aulne, 5 p. 100 seulement pour l'autre traitement.

Les ali!nes ont tous survécu.

Les différences de croissance de l'épicéa entre traitements sont par contre peu marquées, à la limite de la signification, à l’avantage toutefois du traitement comprenant des aulnes.

En fait, la croissance a été faible $(9,2$ et $7,7 \mathrm{~cm}$ respectivement au cours de la seconde année pour les plants non dépérissants) en raison probablement d'un certain déficit du sol en phosphore.

\section{- Nutrition minérale}

Des analyses foliaires effectuées sur l'épicéa à la fin de la période d'engorgement indiquent en seconde année une carence azotée nette dans le traitement ne comportant par daulne : 1,08 p. 100 de $N$ en moyenne, contre 1,45 p. 100 dans lautre traitement. Il n'y a par contre pas de différence sensible entre traitements pour les autres éléments nutritifs.

\section{- Enracinement}

A la fin de l'expérience (automne de la seconde année), des observations ont montré un enracinement «long » plus profond et nettement plus dense, et un bien plus grand nombre de racines courtes (et apparemment mycorhizées) chez l’épicéa en mélange avec laulne que chez l'épicéa pur.

\section{IV. - Discussion et conclusion}

Le caractère néfaste d’une nappe relativement superficielle pour le comportement aérien, l'enracinement, la nutrition azotée de jeunes plants d'épicéa apparaît bien dans cette expérience. Les observations effectuées sont bien en accord avec de précédents résultats (notamment LEVY, 1971 et 1981) et confirment l'intérêt d'un abaissement de la nappe.

Dans cette optique, le mélange réalisé dans cette expérience (5 épicéas pour 1 aulne) fournit un exemple de l'efficacité que l'on peut attendre de l'utilisation de cette dernière espèce, bien que le microclimat de la serre modifie peut-être les différences d’évapotranspiration entre traitements.

Malgré son efficience, on aurait pu craindre que l'aulne ne recommence à effectuer son travail d'assainissement qu'un certain temps après le redémarrage annuel 
de l'activité de lépicéa; les mesures d’évapotranspiration semblent montrer qu'il n'en est rien, ce qui est à mettre en parallèle avec les dates de débourrement des bourgeons terminaux des deux espèces dans cette expérience : l'épicéa a pratiquement 3 semaines de retard, en moyenne, par rapport à l'aulne.

Il est assez probable qu’en forêt l'aulne contribue à l'abaissement de la nappe, non seulement par augmentation de la transpiration de la plantation, mais également par un accroissement du drainage profond, en raison de son exceptionnelle capacité denracinement dans les horizons Bg des pseudogleys (Kreutzer, 1961). Cet abaissement, outre son influence favorable immédiate sur le comportement de l'épicéa (et sans doute d'autres essences de reboisement), permettrait sans doute à moyen terme de lutter efficacement contre la dégradation des propriétés physiques du sol due à la présence périodique d'une nappe superficielle. De plus l'aptitude de l'aulne à fixer l'azote atmosphérique par l'intermédiaire de ses nodosités ne peut qu'améliorer les propriétés chimiques du sol.

Cette expérience montre que l'aulne résiste à un déficit hydrique assez important dans le sol, en été. Il contribue cependant activement à ce dessèchement du sol, en raison sans doute de son enracinement dense et profond dans les pseudogleys. Aussi, le mélange d'aulne et d'épicéa peut-il surtout être conseillé, a priori, dans des stations dont le sol ne se dessèche pas trop en été, en raison par exemple de leur situation topographique. On peut cependant s'attendre à ce que l'assainissement provoqué par l'aulne permette une bien meilleure prospection du sol par les racines d'épicéa, comme cette expérience a pu le montrer, donc favorise l'alimentation estivale en eau de cette dernière espèce.

Il sera utile, sur le plan pratique, de mettre au point une sylviculture des plantations mélangées épicéa-aulne dans des pseudogleys, par exemple de préciser la proportion de chacune des deux espèces et les conditions de taille ou même de recépage de l'aulne, en fonction des diverses conditions écologiques susceptibles d'être rencontrées : fluctuations de la nappe, dessèchement estival du sol.

Il sera bon également de se préoccuper de la provenance des aulnes; en effet, non seulement leur forme et leur vitesse de croissance en dépendent (KosTER, 1971), mais il en est de même du développement de leur système racinaire, les différences pouvant être considérables (SchmidT-VogT, 1971).

\title{
Reçu pour publication en avril 1981.
}

\author{
Summary \\ Influence of Alder (Alnus glutinosa L.) \\ in a mixed Alder-Norway spruce young plantation, \\ on a soil with temporary water-table
}

We have estimated during two years the efficiency of a mixed Alder-Norway Spruce plantation (one Alder for five Spruces) to drain the soil, relatively to a plantation of Spruce alone, in well controlled conditions (greenhouse, vats).

The plants were two years old at the beginning of the experiment. The water-table in the treatment with Spruce alone was kept up near the soil surface by water periodical supplies; an identical volume was supplied to the mixed treatment. 
The mixed treatment (Alder + Spruce) lowers much the water-table; the mean evapotranspiration is 19 to 34 p. 100 greater than for the treatment with Spruce alone, according to the conditions. This effect on water-table starts since the beginning of the annual growth period of Spruce.

This water-table lowering has beneficial effects on Spruce behaviour (rooting, nitrogen nutrition, survival).

In summer. after disappearance of the water-table, the effects of Alder on Spruce, in a mixed plantation, can be opposite : it reduces the soil water content, but the soil volume settled by Spruce rooting is much increased.

\section{Références bibliographiques}

BraUn H.J., 1974. (Amount and seasonal pattern of growth, water consumption and productivity of water consumption in woody species. I. Alnus glutinosa and Salix alba «Liempde»). Allgemeine Forst-u. Jagdzeitung, 145 (5), 81-86.

Delvaux J., 1968. Types de sols, enracinement et production. Observations concernant l'épicéa, les sapins et le douglas. Bull. Soc, r. Belg., n" 1, janvier, 3-6.

Ferda J., 1973. (Improvement of unfavourable microclimatic conditions on waterlogged clearfelled areas by means of amelioration measures). Vedecke Prace Vyskumného Ustava melioraci ve Zbraslavi, $\mathrm{n}^{\circ} 13,299-324$.

(ill. C.J., 1970. The flooding tolerance of woody species. A review. For. Abstr., 31 (4), $671-688$.

GıLL. C.J., 1975. The coological significance of adventitious rooting as a response to flooding in woody species, with special reference to Alnus glutinosa (L.) Gaertn. Flora, G.D.R., 164 (1), 85-97.

Hausser K., 1973. (Fertilizer and amelioration trials in Scots Pine on medium or upper Bunter sandstone in the Schwartzwald in Württemberg. I - Fertilizers trials. II - Amelioration trials). Allgemeine Forst-u. Jagdzeitung, 144 (9/10, 11), 169-186, 205-219.

HeinsidorF D., 1974. (Results of a site amelioration trial with Pinus sylvestris and Quercus rubra on a humus-poor sandy soil in the lowland zone of E. Germany). Beiträge für die Forstwirtschaft, 8 (1, 2). 25-37, 105-110.

Kostik R., 1971. (Juvenile growth of Alder). Polutier, 8 (4), 63-66.

KreUTZER K., 1961. Wurzelbildung junger Waldbäume auf Psendogleyböden. Forstwissen schaftliches Centralblatt, $80(11 / 12), 356-392$.

LeVy G., 1971. Influence de l'engorgement de printemps et de la sécheresse d'été sur le comportement de jeunes plants d'épicéa. Ann. Sci. for., 28 (4), 403-423.

Levy G., 1972. Premiers résultats cuncernants deux expériences d'assainissement du sol sur plantations de résineux. Ann. Sci. for., 29 (4), 427-450.

Ll:vy G., 1978. Nutrition et production de l'épicéa commun adulte sur sols hydromorphes en Lorraine; Iiaisons avec les caractéristiques stationnelles. Amn. Sci. for., 35 (1), 33-53.

Livy G., 1981. Comportement de jeunes plants d'épicéa commun en sol à engorgement temporaire de surface : influence de divers facteurs du milieu. Amn. Sci. for., 38 (1), 3-30.

SCHMIDT-VoGT H., 1971. (Growth and root development in different provenances of Alnus glutinosa in W. Germany). Allgemcine Forst-?t. Jagdzeitung, 142 (6), 149-156. 